\title{
ATLAS Measurements of Isolated Photon Cross-Sections
}

\author{
Marcello Fanti \\ (on behalf of the ATLAS Collaboration)
}

Università di Milano and INFN, Italy

\begin{abstract}
This document presents measurements of the cross-sections for the inclusive production of isolated prompt photons and di-photon events in proton-proton collisions at a centre-of-mass energy $\sqrt{s}=7 \mathrm{TeV}$, performed by the ATLAS experiment at the LHC. Photon candidates are identified by combining information from the calorimeters and from the inner tracker. Residual background in the selected sample is estimated from data based on the observed distribution of the transverse isolation energy in a narrow cone around the photon candidate. The results are compared to predictions from next-to-leading order perturbative QCD calculations.
\end{abstract}

Keywords: photon, di-photon, QCD, cross-section, LHC, ATLAS

PACS: $01.30 . \mathrm{Cc}, 14.70 . \mathrm{Bh}$

Photon production in proton-proton collisions occurs at leading order via quark-gluon scattering, $q g \rightarrow q \gamma$, or quarkantiquark annihilation, $q \bar{q} \rightarrow g \gamma$. The main processes for di-photon production are the quark-antiquark annihilation, $q \bar{q} \rightarrow \gamma \gamma$, and the gluon-gluon interaction, $g g \rightarrow \gamma \gamma$. The latter, mediated by a quark box diagram, is comparable to the former despite the higher power of $\alpha_{s}$, due to the large gluon flux at the LHC. Photons may also be produced at higher order, via initial and final state radiation from quarks, and through parton fragmentation. Therefore, the measurement of photon and di-photon production provides a clean test of the QCD, using a colorless probe which produces a well localized signal in the detector.

This document reports recent measurements of the production cross-sections for inclusive photon [1, 2] and diphoton [3] final states, and comparisons with theoretical next-to-leading order (NLO) evaluations. The data sample consists of the proton-proton collisions recorded in 2010 by the ATLAS experiment, for an integrated luminosity of $\sim 35 \mathrm{pb}^{-1}$. The ATLAS detector is described in Ref [4]. A polar coordinate system is defined, with the polar axis along the proton beam direction at the nominal interaction point, and $\theta$ and $\phi$ defined as the polar and azimuthal angles. The pseudorapidity is defined as $\eta=-\ln [\tan (\theta / 2)]$.

Photon reconstruction and identification are detailed in Ref [5]. A photon produces a narrow shower in the electromagnetic calorimeter (ECAL), detected as a well-localized energy deposit (cluster). If the photon converts into an $e^{+} e^{-}$pair in the material upstream of the ECAL, one or two charged tracks in the inner tracking detectors (ID) may be associated to the cluster. In this case, a careful disambiguation with respect to electrons is needed, based on the possible detection of a displaced vertex and on the ratio between the energy measured in the ECAL and the track momentum from the ID. The residual background from electrons faking photons is estimated from the number of reconstructed $e^{ \pm} \gamma$ pairs with an invariant mass close to that of the $Z$ boson.

The main background to photon detection is due to hadronic jets. They produce broader energy deposits in the ECAL, with a sizable energy leaking into the hadron calorimeter (HCAL) located beyond the ECAL. Jet-photon discrimination is based on these properties, thanks to the 3-dimensional granularity of the ATLAS calorimetry. The ECAL, in particular, is segmented in three longitudinal layers: the middle layer, which collects most of the photon shower energy, is divided into narrow projective cells $(\Delta \eta \times \Delta \phi=0.025 \times 0.025)$, while the front layer consists of very thin strips cells $(\Delta \eta=0.003)$. Most of the jets are rejected by requiring little energy in the HCAL and a narrow shower in the middle layer. The remaining background, due mostly to energetic $\pi^{0}$ mesons decaying into a collimated photon pair, is further reduced by checking whether the shower shape in the strips has two maxima, or has a width not compatible with that of a single photon. These requirements are referred to as photon identification, and photons satisfying them candidates.

The photon identification efficiency ranges between $60 \%$ and $95 \%$, increasing with the transverse energy $E_{\mathrm{T}}^{\gamma}$. This is derived from simulation, after correcting the shower shape distributions to better match those observed in collision data [1]. The achieved jet rejection is $\approx 10^{4}$. Nonetheless, the hadronic background remains sizable. For this reason, data-driven methods are needed to estimate and subtract it.

A powerful discriminant between photons and jets is the isolation energy, $E_{\mathrm{T}}^{\mathrm{iso}}$, defined as the transverse energy 
flow surrounding the photon candidate in a cone of size $\sqrt{\Delta \eta^{2}+\Delta \phi^{2}}<0.4$. The core, containing the photon shower, is excluded, and a correction for the ambient energy is applied event by event [6,7]. A photon is defined as isolated if $E_{\mathrm{T}}^{\text {iso }}<3 \mathrm{GeV}$. This implies a transverse energy flow due to interacting particles $E_{\mathrm{T}}^{\text {iso(part) }}<4 \mathrm{GeV}[1,3]$. The experimental results are therefore presented as fiducial cross-sections for isolated photons.

The $E_{\mathrm{T}}^{\text {iso }}$ distributions for photons and hadronic background are obtained directly from collision data, without relying on simulations. For the background, a control sample is used, defined by reconstructed photons which satisfy the identification criteria in the HCAL and ECAL middle layer, but fail the identification in the ECAL strips. The $E_{\mathrm{T}}^{\text {iso }}$ distribution of genuine photons is then extracted from all photon candidates passing the identification, by subtracting the background component. An independent cross-check is done, using a pure sample of electron candidates from $Z, W^{ \pm}$decays, which behave very similarly to photons in the ECAL: an excellent agreement is found, as shown in Fig. 1. More details are given in Ref [3].
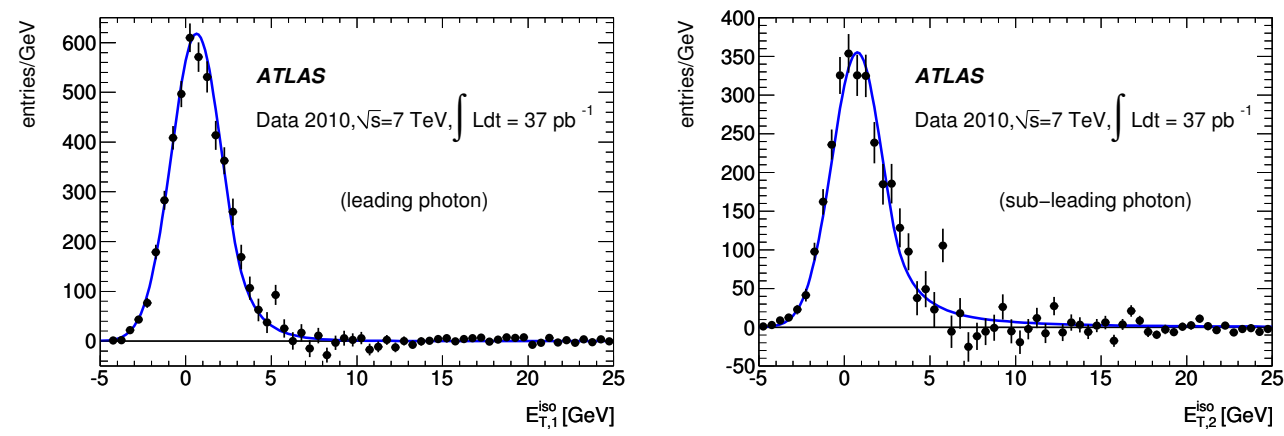

FIGURE 1. Distribution of the isolation energy $E_{\mathrm{T}}^{\text {iso }}$, for the leading (left) and sub-leading (right) photon, extracted from collision data, from identified photons after background subtraction (dots), and from an electron sample (lines).

The signal yield for isolated photons is measured as a function of $E_{\mathrm{T}}^{\gamma}$, in four different pseudorapidity regions, corresponding to the boundaries of the ECAL, or to significant variations of the material distribution in front of it. The background from hadronic jets is subtracted with a "two-dimensional sidebands" technique: a signal region $A$ contains reconstructed photons passing both the identification and the isolation requirement, while three control regions $B, C, D$ collect the reconstructed photons failing respectively the isolation requirement, or the ECAL strips identification, or both. The $E_{\mathrm{T}}^{\text {iso }}$ distribution is essentially independent of the photon identification, and the control regions are dominated by the background, therefore the signal yield $N_{\gamma}$ in region $A$ can be computed from the observed counts in the four regions:

$$
N_{\gamma}=N_{A}-\frac{N_{B} N_{C}}{N_{D}}
$$

The signal purity in region $A$ is higher than $90 \%$ for $E_{\mathrm{T}}^{\gamma}>50 \mathrm{GeV}$.

The observed differential cross-section, as a function of $E_{\mathrm{T}}^{\gamma}$, is shown in Fig. 2. A good agreement is observed with the NLO computation from JETPHOX [8], especially for $E_{\mathrm{T}}^{\gamma}>50 \mathrm{GeV}$. The lower region is more difficult to model, being affected by a larger contribution from fragmentation.
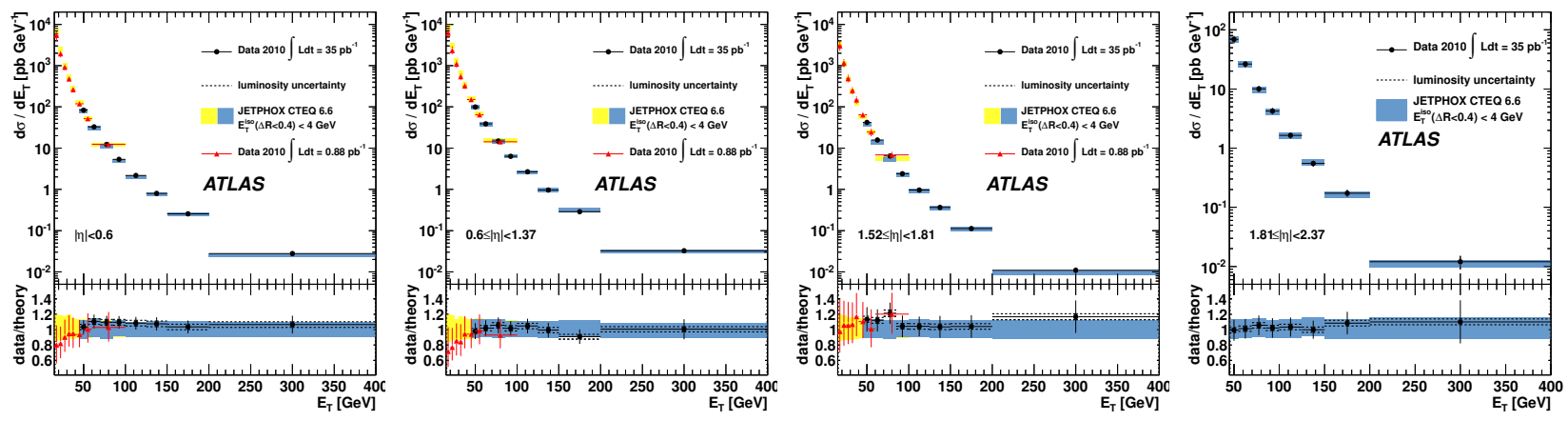

FIGURE 2. Differential cross-section of isolated photons, as a function of $E_{\mathrm{T}}^{\gamma}$, in four different pseudorapidity regions. 
For the di-photon measurement, a base event sample is selected, requiring two reconstructed photons passing the identification criteria. Both photons are tested to pass the isolation requirement, thus having four possible outcomes PP, PF, FP, FF ("P"=pass, "F"=fail), from which four weights $W_{\gamma \gamma}, W_{\gamma \mathrm{j}}, W_{\mathrm{j} \gamma}, W_{\mathrm{jj}}$ are computed:

$$
\left(\begin{array}{l}
\mathrm{PP} \\
\mathrm{PF} \\
\mathrm{FP} \\
\mathrm{FF}
\end{array}\right)=\left(\begin{array}{cccc}
\varepsilon_{1} \varepsilon_{2} & \varepsilon_{1} f_{2} & f_{1} \varepsilon_{2} & f_{1} f_{2} \\
\varepsilon_{1}\left(1-\varepsilon_{2}\right) & \varepsilon_{1}\left(1-f_{2}\right) & f_{1}\left(1-\varepsilon_{2}\right) & f_{1}\left(1-f_{2}\right) \\
\left(1-\varepsilon_{1}\right) \varepsilon_{2} & \left(1-\varepsilon_{1}\right) f_{2} & \left(1-f_{1}\right) \varepsilon_{2} & \left(1-f_{1}\right) f_{2} \\
\left(1-\varepsilon_{1}\right)\left(1-\varepsilon_{2}\right) & \left(1-\varepsilon_{1}\right)\left(1-f_{2}\right) & \left(1-f_{1}\right)\left(1-\varepsilon_{2}\right) & \left(1-f_{1}\right)\left(1-f_{2}\right)
\end{array}\right)\left(\begin{array}{l}
W_{\gamma \gamma} \\
W_{\gamma \mathrm{j}} \\
W_{\mathrm{j} \gamma} \\
W_{\mathrm{jj}}
\end{array}\right)
$$

The quantities $\varepsilon_{i}$ and $f_{i}$ are the probabilities that a genuine photon or a fake photon pass the isolation cut, for each of the two photon candidates. Since sizable correlations between the two photon candidates are observed in data, the matrix actually used in the analysis is more complex than that in Eq. (2), involving also conditional probabilities. All matrix coefficients are derived from the isolation energy distributions, extracted from collision data. The signal yield of isolated di-photon events is then computed as a weighted sum over the base di-photon sample:

$$
N_{\gamma \gamma}=\sum_{k}\left[\varepsilon_{1} \varepsilon_{2} W_{\gamma \gamma}\right]_{(k-\text { th event })}
$$

The purity of the sample is $\sim 65 \%$.

The differential cross-section, as a function of the di-photon azimuthal separation $\left(\Delta \phi_{\gamma \gamma}\right)$, invariant mass $\left(m_{\gamma \gamma}\right)$, and transverse momentum $\left(p_{\mathrm{T} \gamma \gamma}\right)$, is shown in Fig. 3. The experimental result is compared with two NLO computations, from DIPHOX [9] and ResBos [10]. The $\Delta \phi_{\gamma \gamma}$ distribution is broader at lower values than expected from theory: this has already been observed in proton-antiproton collisions at the Tevatron. As a consequence, a deviation at low invariant masses $\left(m_{\gamma \gamma}<30 \mathrm{GeV}\right)$ is also observed. Apart for this issue, the experimental results are in good agreement with the theoretical prediction.
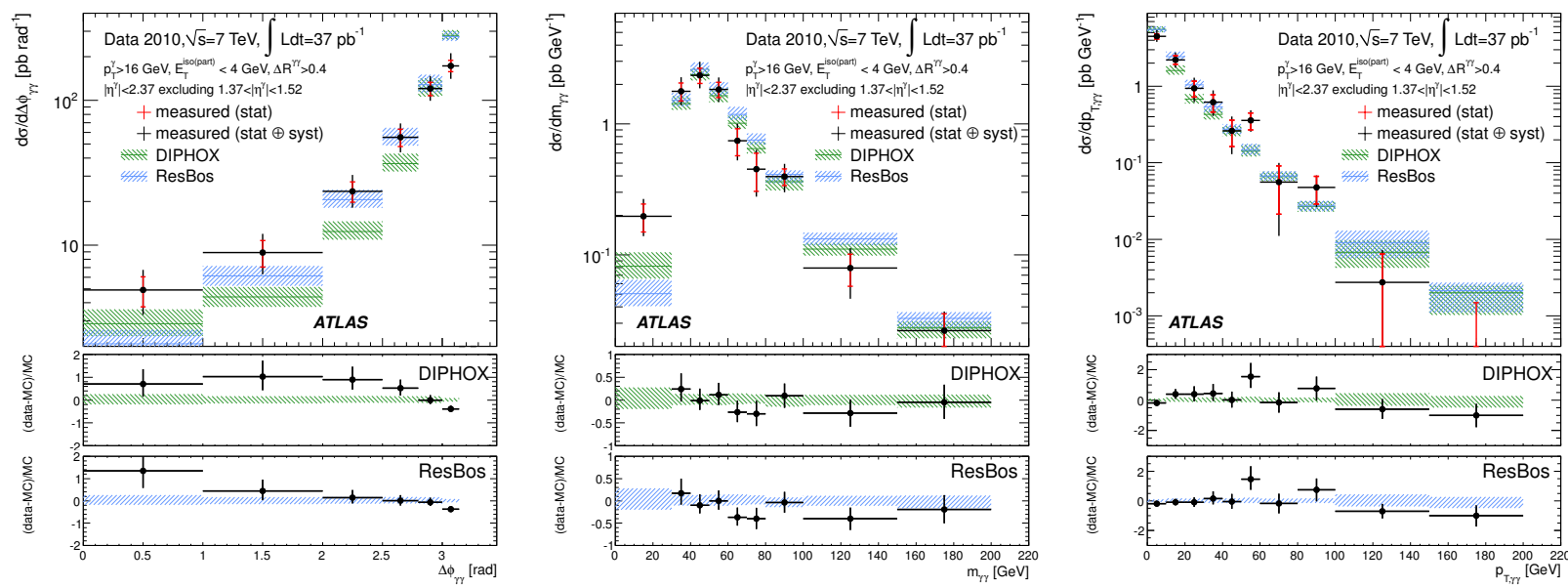

FIGURE 3. Differential cross-section of di-photon production, as a function of the di-photon azimuthal separation $\left(\Delta \phi_{\gamma \gamma}\right)$, invariant mass $\left(m_{\gamma \gamma}\right)$, and transverse momentum $\left(p_{\mathrm{T} \gamma \gamma}\right)$.

\section{REFERENCES}

1. ATLAS Collaboration, Phys. Rev. D83, 052005 (2011).

2. ATLAS Collaboration, $\operatorname{arXiv}(2011), 1108.0253 \mathrm{v} 1$.

3. ATLAS Collaboration, $\operatorname{arXiv}(2011), 1107.0581 \mathrm{v} 1$.

4. ATLAS Collaboration, JINST 3, S08003 (2008).

5. ATLAS Collaboration, ATL-PHYS-PUB-2011-007 (2011), URL http: / / cdsweb. cern . ch/record/1345329.

6. M. Cacciari, G. P. Salam, and S. Sapeta, JHEP 04, 065 (2010).

7. M. Cacciari, G. P. Salam, and G. Soyez, JHEP 04, 005 (2008).

8. S. Catani, and others, JHEP 05, 028 (2002).

9. T. Binoth, J. Guillet, E. Pilon, and M. Werlen, Eur. Phys. J. C16, 311-33 (2000).

10. C. Balazs, E. L. Berger, P. M. Nadolsky, and C. P. Yuan, Phys. Rev. D76, 013009 (2007). 